\title{
Characterization of Capsaicin-Loaded Nanoemulsions Stabilized with Alginate and Chitosan by Self-assembly
}

\author{
Ae-Jin Choi • Chul-Jin Kim • Yong-Jin Cho • \\ Jae-Kwan Hwang • Chong-Tai Kim
}

Received: 1 October 2010 /Accepted: 18 March 2011 / Published online: 6 April 2011

(C) The Author(s) 2011. This article is published with open access at Springerlink.com

\begin{abstract}
The objectives of this research were to characterize capsaicin-loaded nanoemulsions stabilized with natural biopolymer such as alginate and chitosan for use as a functional ingredient delivery system. The biopolymer nanoemulsion was prepared using self-assembly emulsification methods, and the capsaicin included oleoresin capsicum as the core material in the nanoemulsion. The particle sizes of the double-layer nanoemulsions prepared with alginate (AN) and chitosan $(\mathrm{CN})$ were $20 \mathrm{~nm}$ or lower. The triple-layer nanoemulsion, which was prepared by complexation with chitosan and alginate (CAN), was successfully prepared with particle sizes on the nanoscale through the electrostatic interactions between the carboxylic groups and amine groups of the biopolymers. The Zeta potential value, which is an indicator of the overall stability and physicochemical properties of the nanoemulsion, of AN and $\mathrm{CN}$ were significantly higher than $\mathrm{SN}$ and CAN $(p<0.01)$. In conclusion, the double-layer nanoemulsions incorporated with alginate and chitosan can be expected to improve the stability of nanoemulsion. In addition, this system holds promise for use in the production of functional foods containing functional ingredients.
\end{abstract}

C.-J. Kim · Y.-J. Cho $\cdot$ C.-T. Kim $(\bowtie)$

Bio-Nano Research Center, Korea Food Research Institute,

Seongnam, Kyeonggi 463-746, South Korea

e-mail: ctkim@kfri.re.kr

A.-J. Choi

Department of Herbal Crop Research,

National Institute of Horiculture \& Herbal Science, RDA,

Chungbuk 369-873, South Korea

J.-K. Hwang

Department of Biotechnology, Yonsei University,

Seoul 120-749, South Korea
Keywords Food nanoemulsion · Self-assembly emulsification · Oleoresin capsicum · Biopolymer

\section{Introduction}

The potential benefits of nanotechnology have been recognized by many industries and commercial products based on nanotechnology are already being manufactured in the microelectronics, aerospace, and pharmaceutical industries. In contrast, applications of nanotechnology in the food industry are rather limited (Chen et al. 2006a, b) However, nanotechnology has the potential to impact many aspects of food and agricultural systems. Food security, disease treatment delivery methods, new tools for molecular assays, and protection of the environment are examples of where nanotechnology can be applied to agriculture and food systems. Major areas in food production may benefit from nanotechnology, which include the development of new functional materials, microscale and nanoscale processing, product development, and methods and instrumentation design for improved food safety and biosecurity (Weiss et al. 2006).

One of the most promising nanotechnologies is the nanoemulsion delivery system, which is being used to enhance the stability and the oral bioavailability of lipophilic functional agents (Wang et al. 2007). A wide variety of different systems have been developed to encapsulate lipophilic functional agents, including lipid vehicles such as oil, surfactant dispersions, association colloids, microemulsions, nanoemulsions, self-emulsifying formulations, emulsions, liposomes, and so on (McClements et al. 2007). Nanotechnology has shown greater potential in improving the efficiency of delivery of nutraceuticals and 
bioactive compounds in functional foods to improve human health (Neethirajan and Jayas 2011).

Oil-in-water $(\mathrm{O} / \mathrm{W})$ emulsions have been used as vehicles for the delivery of lipophilic bioactives in the food and drug industry. However, food emulsions are thermodynamically unstable systems and will eventually breakdown due to the increase in interfacial area after emulsification. Consequently, the physical instability of the emulsion results in creaming, flocculation, coalescence, phase inversion, and Ostwald ripening. Simple solutions or nanoemulsions as food delivery systems may be unstable or degradable due to changes in the nature of the emulsion structure and environmental conditions such as temperature, light, and $\mathrm{pH}$. However, it is possible to develop nanoparticles as novel delivery systems using food-grade biopolymers such as polysaccharides (alginate, chitosan, pectin, gum arabic) and proteins (casein, gelatin, whey) by promoting self-association or aggregation of single biopolymers or by inducing phase separation in mixed biopolymer systems (Weiss et al. 2006). These biopolymers have been used in nanocomposite films formed by biopolymer matrix reinforced with nanoparticles (Voon et al. 2010).

Recently, nanoemulsion technology has been successfully applied as nano-ingredients in functional food such as capsaicin and resveratrol (Kim et al. 2008; Cho et al. 2008). The bioactive ingredient can be encapsulated in nanometersized structures, which function as the transport systems. These nanometer-sized structures have sizes ranging from 20-500 $\mathrm{nm}$ and provide the necessary physical properties, such as solubility, stability, and bioavailability (Choi et al. 2008). In this study, a nanopolymeric system was applied to the formulation of nanoemulsions using self-assembly emulsification for the development of a bioactive ingredient delivery system, and the structure and stability of the biopolymer-based nanoemulsions were characterized. The core materials consisted of oleoresin capsicum (OC), which was successfully used for the fabrication of nanoemulsions in our previous studies. The stability of bioactive ingredients in nanoemulsions coated with a biopolymer such alginate and chitosan were expected to increase, allowing for long-term storage.

\section{Materials and Methods}

\section{Materials}

Oleoresin capsicum (OC, SHU 100,000) was supplied by G\&F (Seoul, Korea). Polyoxyethylene sorbitan monooleate (Tween 80) was purchased from Sigma Chemical Co. (St. Louis, MO, USA). Capsaicinoid was purchased from Fluka (capsaicin/dihydrocapsaicin=65:35, Buchs SG, Switzerland).
Chitosan was prepared by demineralization, deproteinization, and deacetylation from crab shell. Next, the chitosan (degree of deacetylation $93 \%$, molecular weight 330,000 ) was dissolved in a mixed aqueous solution of $20 \%$ hydrochloride solution and distilled water, after which the mixture was adjusted to $\mathrm{pH}=5.6$ and stirred for $3 \mathrm{~h}$. The reaction mixture was then subjected to a continuous membrane filtration process, after which a permeate was freeze-dried (Seo and Toshio 2002). Alginate sodium salt(from brown algae, $\sim 3,500 \mathrm{cp}, 2 \%, 25^{\circ} \mathrm{C}$ ) was purchased from Sigma Chemical Co. (St. Louis, MO, USA).

All other reagents were of analytical grade. Deionized distilled water was purchased from Waters (Milford, MA, USA).

\section{Preparation of Chitosan/Alginate-Based Nanoemulsions}

Chitosan/alginate nanoemulsions were prepared using the self-assembly method. The schematic representation of formation of multilayer emulsions and preparation procedure of nanoemulsions based on alginate/chitosan were shown in Figs. 1 and 2, respectively. Single-layer nanoemulsion is formed by stirring oil and aqueous phases together in the presence of a water-soluble emulsifier. Double-layer nanoemulsions are formed by coating singlelayer nanoemulsion with biopolymer. Triple-layer nanoemulsion is formed by adding polyelectrolytes to an emulsion containing oppositely charged biopolymer, such as anion-charged alginate and cation-charged chitosan.

Nanoemulsions are classified into three groups: singlelayer nanoemulsion (SN) based on the aqueous phase with water, double-layer nanoemulsion $(\mathrm{AN}, \mathrm{CN})$ based on alginate and chitosan, and triple-layer nanoemulsion (CAN) based on chitosan/alginate. The nanoemulsions were prepared by self-assembly emulsification according to the following procedure. Briefly, the chitosan/alginate-based nanoemulsions were prepared using Tween 80 , which was selected as the optimum detergent for the formation of $\mathrm{O} / \mathrm{W}$ nanoemulsion containing oleoresin capsicum by pseudoternary phase diagram. The optimum condition of the oil phase-surfactant for the production of chitosan/alginate-based $\mathrm{O} / \mathrm{W}$ nanoemulsions was determined to be an OC/Tween 80 ratio of 1:3. The alginate and chitosan solution was prepared at a concentration of $0.05 \%$ in aqueous phase to produce $\mathrm{AN}$, $\mathrm{CN}$ and CAN under constant stirring, and the polymer solutions was then heated up to $100{ }^{\circ} \mathrm{C}$ for $5 \mathrm{~min}$ with heating and stirring plate and cooled down to room temperature. To prepare the double-layer nanoemulsions, $\mathrm{AN}$ and $\mathrm{CN}(\mathrm{OC} /$ Tween $80=1: 3,0.5 \mathrm{~g}$ ) were mixed for $5 \mathrm{~min}$ at $25^{\circ} \mathrm{C}$ under constant stirring. After this, $100 \mathrm{ml}$ of aqueous phases $(0.5 \%$, $w / v)$ were gradually added to the oil phase and surfactant with constant stirring using a laboratory mixer for $2 \mathrm{~h}$ at $25^{\circ} \mathrm{C}$, respectively. Chitosan/alginate-based nanoemulsions were 
Fig. 1 Schematic representation of formation of single- and multilayer emulsions

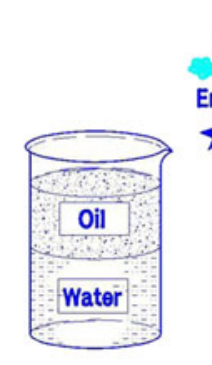

Mixing \& Stirring stabilized at $25{ }^{\circ} \mathrm{C}$ for $24 \mathrm{~h}$ and then filtered using a $0.45-\mu \mathrm{m}$ polyvinylidene fluoride (PVDF) membrane filter (Whatman, England).

To prepare the triple-layer nanoemulsion, CAN, the $\mathrm{pH}$ of the $0.05 \%$ alginate and chitosan solutions was initially set to 4.9 and 4.6, respectively. The optimal formulation of the oil phase-surfactant mixture $(0.6 \mathrm{~g})$ was added to $117.5 \mathrm{ml}$ of the $0.05 \%$ alginate solution in a beaker and gently mixed using a stirrer for $30 \mathrm{~min}$. To produce an alginate pre-gel, $7.5 \mathrm{ml}$ of an $18 \mathrm{mM}$ calcium chloride solution was added dropwise for $60 \mathrm{~min}$ under gentle stirring into a beaker. Then, $25 \mathrm{ml}$ of the $0.05 \%$ chitosan solution was added dropwise into the pre-gel over $90 \mathrm{~min}$. A colloidal dispersion at $\mathrm{pH} 4.7$ formed upon polycationic chitosan addition. The chitosan/alginate-based nanoemulsion was stirred for $30 \mathrm{~min}$ to improve curing. The supernatant was collected by centrifugation at $8,000 \times g$ for $45 \mathrm{~min}$ at $4{ }^{\circ} \mathrm{C}$ and filtered with $0.45-\mu \mathrm{m}$ PVDF filter membrane, and then stabilized for $24 \mathrm{~h}$. The resulting

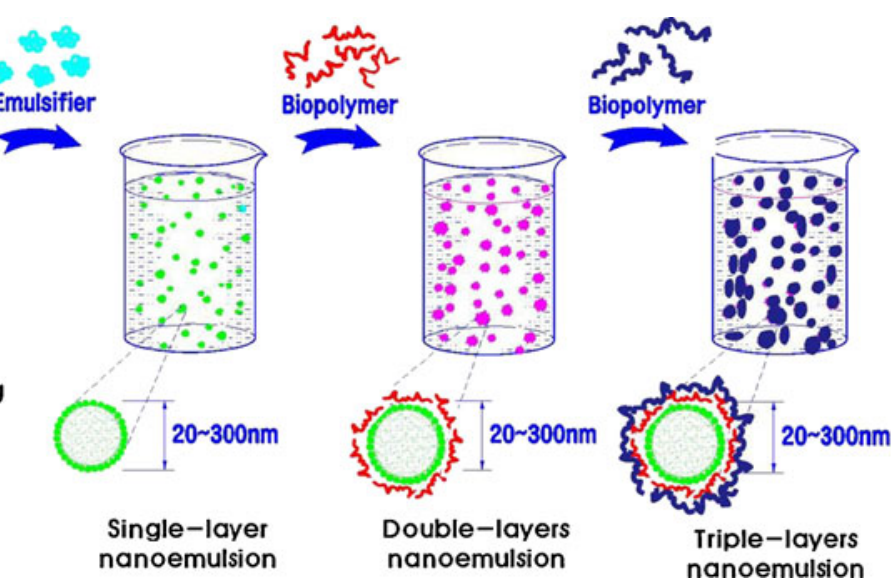

nanoemulsions were then characterized and the stability analyzed.

Determination of Biopolymer Concentration for Preparing Nanoemulsions

The nanoemulsions containing chitosan were manufactured at chitosan concentrations ranging from $0.05-1.0 \%(w / v)$ to determine the optimum concentration of alginate and chitosan for preparing the double-layer nanoemulsions. These nanoemulsions were heated for $15 \mathrm{~min}$ at $75^{\circ} \mathrm{C}$ in water bath, and the stability of the particle size and viscosity was determined (Fig. 3). In these experiments, the optimum concentration of chitosan for preparing the double-layer nanoemulsions was selected based on the particles that displayed the best stability of particle size and viscosity. The viscosity of the biopolymer nanoemulsions was determined without dilution using Brookfiled DV-II+ (Brookfiled Engineering Laboratory Inc., MA,
Fig. 2 Preparation of single, double- and triple- layers nanoemulsions fabricated with $0.05 \%$ alginate and chitosan. $O C$ oleoresin capsicum, $S N$ single-layer nanoemulsion based on aqueous phase, $C N$ doublelayer nanoemulsion fabricated with chitosan, $A N$ double-layer nanoemulsion fabricated with alginate, $C A N$ triple-layer nanoemulsion fabricated with chitosan/alginate

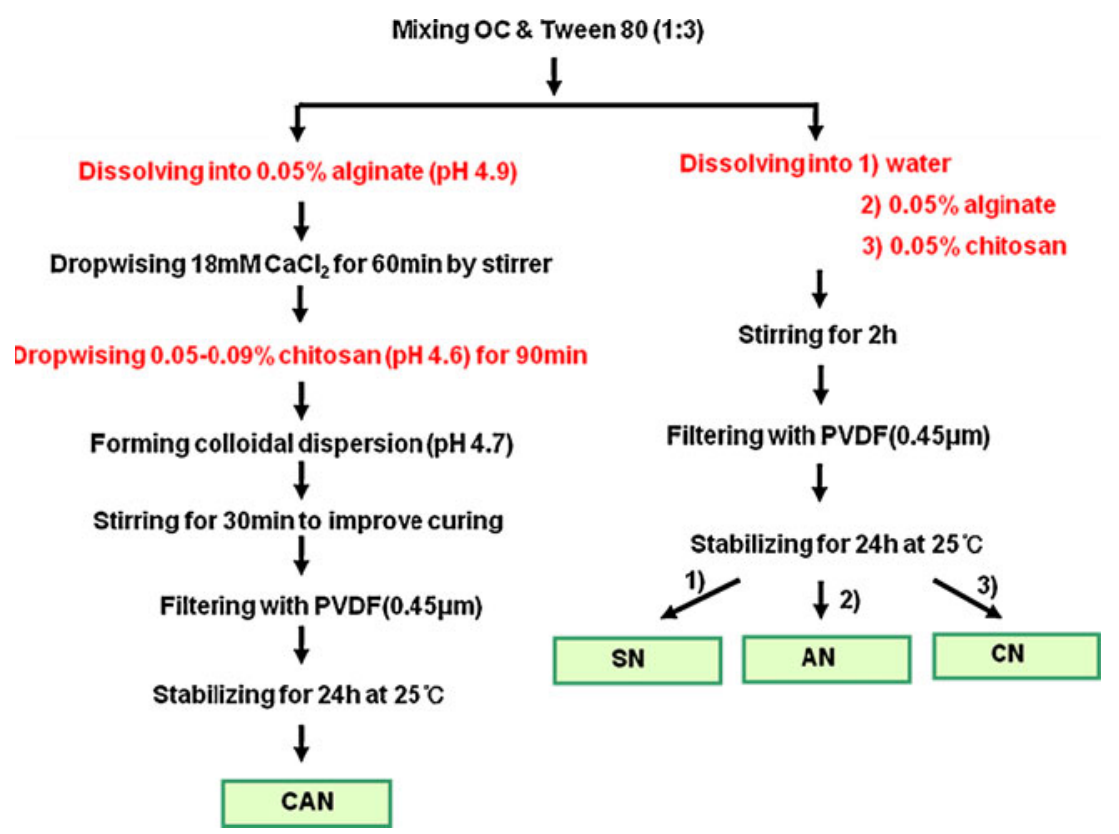



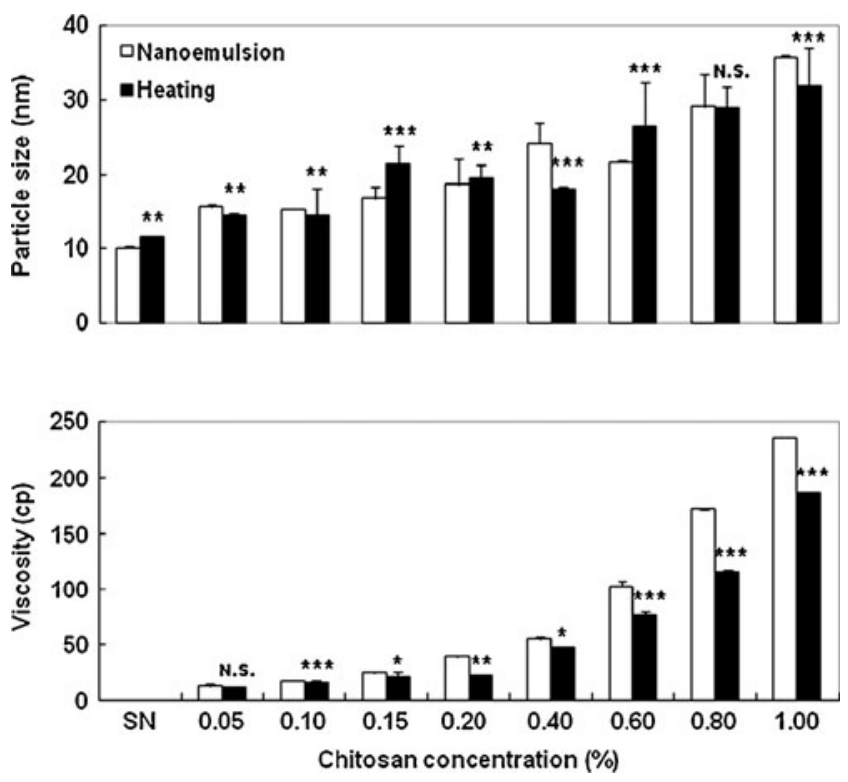

Fig. 3 Effect of chitosan concentration on the particle size and viscosity of $\mathrm{CN}$. $C N$ double-layer nanoemulsion fabricated with chitosan. Results were expressed as the mean \pm SEM $(n=3)$. N.S. not significant. $* P<0.05, * * P<0.01, * * * P<0.001$, significantly different

USA) with spindle 2 at $25{ }^{\circ} \mathrm{C}$. The viscosity was measured after the reaction at $20 \mathrm{rpm}$ for $1 \mathrm{~min}$, and the $\mathrm{cP}$ value was determined at a range of over $10 \%$ the torque value.

To determine the optimum concentration of the alginate and chitosan solution for preparing the triple-layer nanoemulsions, biopolymer nanoemulsions were manufactured by fixing the alginate concentration at $0.05 \%(w / v)$ and varying the chitosan concentration between $0.05 \%$ and $0.09 \%(w / v)$. The stability of the particle size during storage over 7 days at $25{ }^{\circ} \mathrm{C}$ was then determined for each nanoemulsion. The prepared nanoemulsions were centrifuged at $8,000 \times g$ for $45 \mathrm{~min}$, and the precipitates were not involved in the formation of the nanoemulsion. The samples were then dried at $105{ }^{\circ} \mathrm{C}$ for $4 \mathrm{~h}$. The incorporation efficiency was calculated from the ratio of the weight of dried precipitates to the initial weight used to prepare the nanoemulsions. The concentration of alginate and chitosan used for preparing the triple-layer nanoemulsions was selected based on the concentrations that resulted in the best stability of particle size and the highest incorporation efficiency.

\section{Characterization of Biopolymer Nanoemulsions}

\section{Morphology}

The morphology of the biopolymer nanoemulsions was examined in a transmission electron microscope (TEM; Phillips TECNAI 12, Eindhoven, The Netherlands). For negative staining, $7 \mu \mathrm{l}$ of the annealed nanoemulsions were placed on carbon-coated copper grids and washed once with ultrapure water. Then, the samples were stained immediately with $7 \mu \mathrm{l}$ of $2 \%$ uranyl acetate in ultrapure water, washed with water again, and then air-dried. Images were acquired at $120 \mathrm{kV}$, and the scales were calibrated using a grating replica $3 \mathrm{~mm}$ grid. A BioScan camera (Model 792, GATAN, Bioscan Ltd., Washington DC, USA), and Digital Micrograph software (Gatan v 3.4) was used to perform the image capture.

\section{Particle Size Analysis}

Particle size and size distribution of the nanoemulsion were determined by photon correlation spectroscopy, which analyzes the fluctuations in light scattering due to the Brownian motion of the particles (Attwood et al. 1992), using a Nanotrac TM250 (Microtrac Inc., PA, USA). Light scattering was monitored at $25^{\circ} \mathrm{C}$ and an angle of $90^{\circ}$.

\section{Electrophoretic Mobility}

The zeta potential is often a better representation of the electrical characteristics of an emulsion droplet because it inherently accounts for the adsorption of any charged counter ions (McClements et al. 2007).

The zeta potential of nanoemulsion preparations was determined by placing $1 \mathrm{ml}$ of the sample in a disposable zeta cuvette $(1 \times 1 \times 4 \mathrm{~cm})$. The cuvette was inserted into the measurement chamber of the particle electrophoresis instrument (Zetasizer Nanoseries ZS, Malvern Instrument, Worcestershire, UK) and equilibrated to $25{ }^{\circ} \mathrm{C}$ in the Peltier-controlled cuvette holder 2 min before use. The zeta potential was then determined by measuring the direction and velocity that the biopolymer nanoemulsion moved in the applied electric filed at $633 \mathrm{~nm}$ for $1 \mathrm{~min}$. The Smoluchowsky mathematical model was used to convert the electrophoretic mobility measurements into zeta potential values. To ensure that the presence of the buffer did not influence the zeta potential measurements, biopolymer nanoemulsions were initially dispersed in solutions containing a range of different buffer concentrations. The

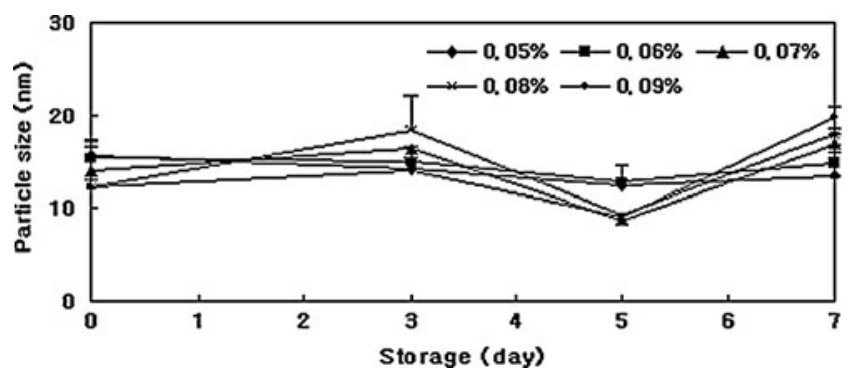

Fig. 4 Effect of chitosan concentration on the particle size of CAN with $0.05 \%$ alginate during storage for 7 days. CAN triple-layer nanoemulsion fabricated with chitosan/alginate. Results were expressed as the mean \pm SEM $(n=3)$ 


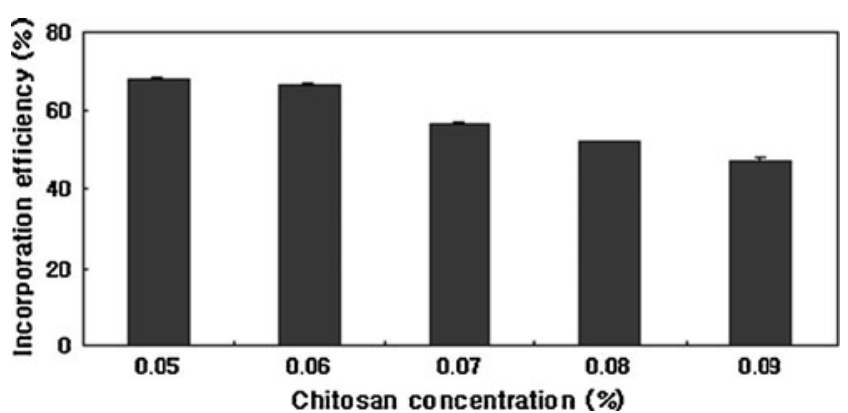

Fig. 5 Incorporation efficiency of CAN at different chitosan concentrations with $0.05 \%$ alginate. $C A N$ triple-layer nanoemulsion fabricated with chitosan/alginate. Results were expressed as the mean $\pm \mathrm{SEM}$ $(n=3)$

results of these experiments indicated that the zeta potential was independent of buffer concentration (data not shown).

\section{Statistical Analysis}

All values were reported as mean \pm SEM from at least four independent experiments. The statistical differences among the groups were tested using student's $t$ test, with more than two groups. ANOVA was used to compare results. Experimental results were considered statistical significant at $95 \%$ confidence (i.e., $p<0.05$ ).

\section{Results and Discussion}

Screening of Biopolymer Concentration for Preparing Nanoemulsions

An increase in the concentration of chitosan from $0.05 \%$ to $1.0 \%$ led to an increase in the particle size and the viscosity of the chitosan-based double-layer nanoemulsions $(p<$ $0.001)$. Fabrication of the double-layer nanoemulsion using the $0.05 \%$ chitosan solution as the aqueous phase resulted in the formation of particles with a minimum size of $10.07 \pm$ $0.34 \mathrm{~nm}$ (Fig. 4). The particle size of the double-layer nanoemulsion based on the $0.05 \%, 0.15 \%, 0.2 \%$, and $0.6 \%$ chitosan solutions was significantly increased by heat treatment at $70{ }^{\circ} \mathrm{C}(p<0.01)$. In addition, the viscosity of the nanoemulsions increased with an increase in the concentration of chitosan. In contrast, no significant change was observed for the nanoemulsions fabricated using the $0.05 \%$ chitosan solution as the aqueous phase after heat treatment $(p<0.05)$. The viscosity rose with an increase in polymer concentration, particularly for high molecular weight samples. Although there were interactions between polymer chains, hydrogen bonds, electrostatic bonds, and hydrophobic interactions, the molecular weight was the main factor affecting the viscosity of chitosan ( $\mathrm{Li}$ et al. 2007). Therefore, the optimum concentration of alginate and chitosan for the preparation of the double-layer nanoemulsions was determined to be $0.05 \%$ after heat treatment.

To determine the optimal biopolymer concentration for preparing the triple-layer nanoemulsions, the nanoemulsions were manufactured at chitosan concentrations ranging from $0.05 \%$ to $1.0 \%$ with an alginate concentration of $0.05 \%$. The particle size of the triple-layer nanoemulsions after fabrication did not vary significantly at different chitosan concentrations and ranged from $12.21 \pm 0.76$ to $15.67 \pm 0.82 \mathrm{~nm}$. The particle size of the nanoemulsions slightly increased after 7 days of storage depending on the concentrations of chitosan $(0.07 \%, 0.08 \%$, and $0.09 \%)(p<$ 0.01 , Fig. 4), which may have been due to the noise in the particle size analysis.

The triple-layer nanoemulsions manufactured using $0.05 \%$ chitosan and $0.05 \%$ alginate solutions had the highest incorporation efficiency, $68.00 \pm 0.34 \%$, and the incorporation efficiency significantly decreased when the concentration of chitosan was higher than $0.05 \%(p<0.001$, Fig. 5). Therefore, the chitosan and alginate concentration that resulted in the highest incorporation efficiency and storage stability was determined to be $0.05 \%$. The high incorporation efficiency of the triple-layer nanoemulsions that occurred because of the polycationic nature of chitosan leads to a strong interaction with negatively charged alginate. When alginate was dropped into the chitosan solution, the electrostatic interaction between the carboxylic groups of alginate and the amine groups of chitosan resulted in the formation of a membrane (GåserØd et al. 1998, 1999).

\section{Characterization of Biopolymer Nanoemulsions}

\section{Difference in Appearance}

The single-, double-, and triple-layer nanoemulsions fabricated using $0.05 \%$ chitosan and $0.05 \%$ alginate solutions

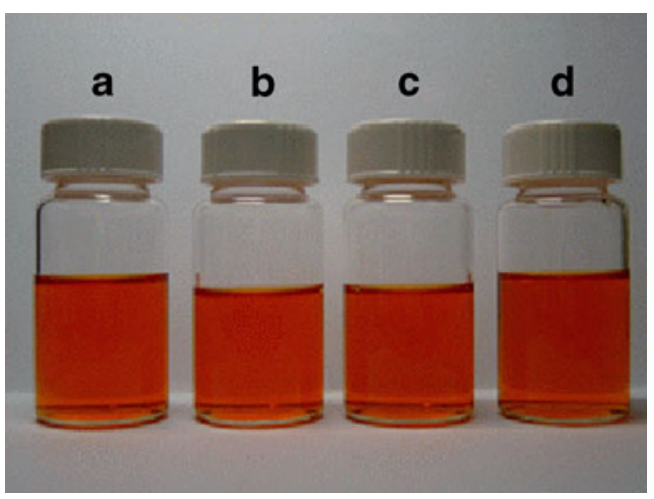

Fig. 6 Difference in appearance of nanoemulsions: $a$ single-layer nanoemulsion ( $\mathrm{SN}$ ) based on aqueous phase; $b$ double-layer nanoemulsion $(\mathrm{CN})$ fabricated with chitosan, $c$ double-layer nanoemulsion (AN) fabricated with alginate, and $d$ triple-layer nanoemulsion (CAN) fabricated with chitosan/alginate 
Fig. 7 TEM of single-, doubleand triple-layer nanoemulsions fabricated with chitosan and alginate. a Single-layer nanoemulsion (SN) based on aqueous phase; $\mathbf{b}$ double-layer nanoemulsion $(\mathrm{CN})$ fabricated with chitosan, c double-layer nanoemulsion (AN) fabricated with alginate, and $\mathbf{d}$ triple-layer nanoemulsion (CAN) fabricated with chitosan/alginate
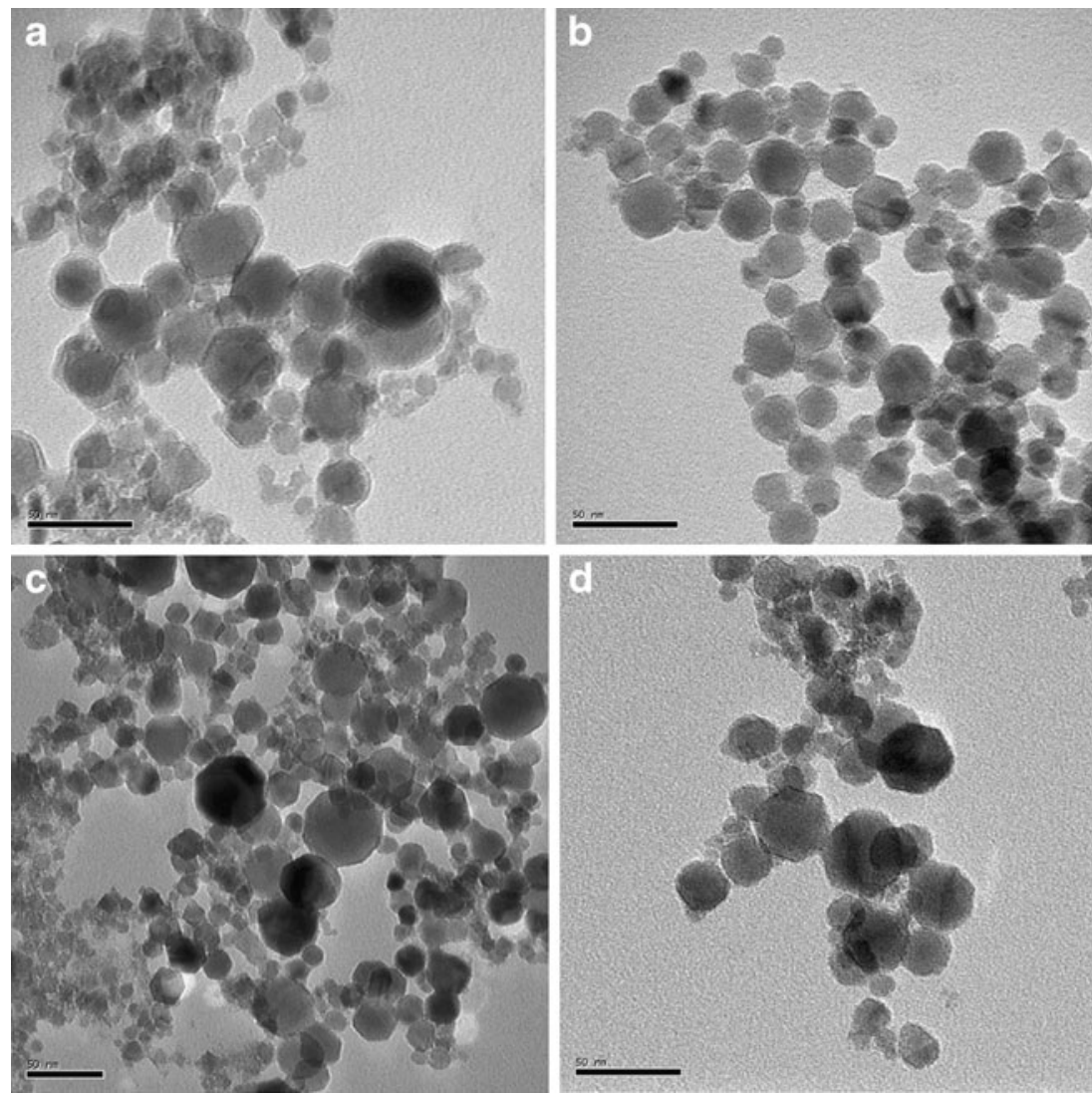

appeared transparent and easily flowed as an oil-in-water $(\mathrm{O} / \mathrm{W})$ nanoemulsion, indicating that the nanoemulsions contained particle sizes below $100 \mathrm{~nm}$ (Fig. 6). Due to the red color and transparent characteristics of the nanoemulsions, biopolymer nanoemulsions formed with OC containing carotenoid components can be used as functional products in the beverage industry to satisfy the personal taste of customers.

\section{Morphology}

The morphological properties of the biopolymer nanoemulsions fabricated using chitosan and alginate were

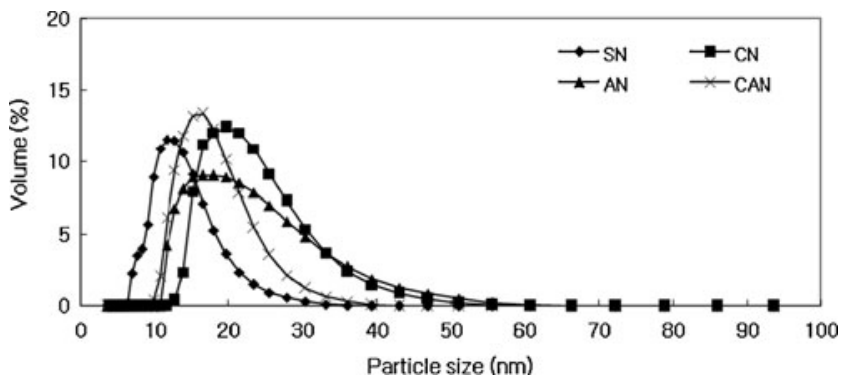

Fig. 8 Particle size distribution of nanoemulsions. $S N$ single-layer nanoemulsion based on aqueous phase, $C N$ double-layer nanoemulsion fabricated with chitosan, $A N$ double-layer nanoemulsion fabricated with alginate, $C A N$ triple-layer nanoemulsion fabricated with chitosan/alginate imaged by TEM (Fig. 7). The TEM images indicate the presence of a droplet-type nanoemulsion structure, which existed in the single-, double-, and triple-layer forms. The hydrophilic portions of the droplets were stained black, while the hydrophobic components were unstained and had a size range between 25 and $70 \mathrm{~nm}$. SN, the single layer nanoemulsion solution, was not uniform in shape and was partially bound to large or small emulsion drops. The CN, AN, and CAN double- or triple-layer nanoemulsions were trapped within the charged biopolymers,

Table 1 Zeta potential, polydispersity, and $\mathrm{pH}$ of nanoemulsions

\begin{tabular}{llll}
\hline & Polydispersity index & Zeta potential $(\mathrm{mV})$ & \multicolumn{1}{c}{$\mathrm{pH}$} \\
\hline $\mathrm{SN}$ & $0.47 \pm 0.01 \mathrm{~b}$ & $-14.2 \pm 1.96 \mathrm{bc}$ & $5.24 \pm 0.12 \mathrm{a}$ \\
$\mathrm{CN}$ & $0.63 \pm 0.12 \mathrm{a}$ & $26.3 \pm 12.7 \mathrm{ab}$ & $4.49 \pm 0.12 \mathrm{~b}$ \\
$\mathrm{AN}$ & $0.39 \pm 0.08 \mathrm{~b}$ & $-31.7 \pm 0.36 \mathrm{a}$ & $5.65 \pm 0.25 \mathrm{a}$ \\
$\mathrm{CAN}$ & $0.66 \pm 0.01 \mathrm{a}$ & $-8.97 \pm 0.43 \mathrm{c}$ & $5.41 \pm 0.43 \mathrm{a}$ \\
$F$-value & $10.02^{*}$ & $8.05^{*}$ & $11.23^{*}$
\end{tabular}

Results were expressed as the mean $\pm \operatorname{SEM}(n=3)$. Means with different letters in the same column differ significantly by Duncan's multiple range test $(p<0.05)$

$S N$ single-layer nanoemulsion based on aqueous phase, $C N$ double-layer nanoemulsion based on chitosan, $A N$ double-layer nanoemulsion based on alginate, $C A N$ triple-layer nanoemulsion based on chitosan/alginate $* P<0.01$, significantly different 
chitosan, alginate, or both of them, respectively. CN, AN, and CAN showed a more compact and clear particle structure, which may be due to flocculation and coalescence caused by phase separation or stabilization of nanoemulsions.

\section{Distribution of Particle Size}

The distribution of particle size, one of the most important physical characteristics of a nanoemulsion, depended on the volume of the particles (Fig. 8). The distribution of particle sizes was more broad for the double-layer nanoemulsions, $\mathrm{AN}$ and $\mathrm{CN}$, than the single- and triple-layer nanoemulsions, SN and CAN. This most likely occurred due to the coating of the highly polymerized compounds, alginate and chitosan.

\section{Zeta Potential and Polydispersity Index}

The droplets in most nanoemulsions have an appreciable electrical charge, and therefore, electrostatic interactions may play an important role in determining their overall stability and physicochemical properties (Nielloud and Marti-Mestres 2000). The zeta potential represents the electric charge between the shear plane of the final external layer and the bulk solution, and this parameter has been used to estimate the property of dispersoids. This factor is influenced by the composition of the nanoemulsions and the electrical phenomenon. Statistically significant differences in the zeta potential among SN, $\mathrm{AN}, \mathrm{CN}$, and CAN prepared with different biopolymers were observed $(p<0.01$, Table 1$)$. The zeta potential of SN was $-14.23 \pm 1.96 \mathrm{mV}$, and the zeta potential of $\mathrm{CN}$ was $26.33 \pm 12.68 \mathrm{mV}$ at $25^{\circ} \mathrm{C}$ due to the positive charge of the chitosan molecules coated on the surface of $\mathrm{CN}$. In contrast, the zeta potential of AN was $-31.70 \pm 0.36 \mathrm{mV}$ due to the negative charge of the alginate molecules. The zeta potential of CAN was $-8.97 \pm 0.43 \mathrm{mV}$, which was lower than SN. This finding suggests that the carboxyl group of alginate interacts with the amine group of chitosan, reducing the number of positive charges exposed on the nanoemulsion surface. These results confirmed that an alginate and chitosan layer was formed on the nanoemulsion surface. The absolute value of the zeta potential can be used as a measure of the stability of the nanoemulsions; therefore, these results confirmed that $\mathrm{CN}$ and AN were comparatively more stable than SN. However, due to the coexistence of anions and cations by bonding with deposited electrical ions, the stability of CAN was lower than SN, CN and AN. The polydispersity index of $\mathrm{SN}$ and $\mathrm{AN}$ was significantly lower than $\mathrm{CN}$ and CAN $(p<0.01)$. Based on these combined results, AN was determined to be the most stable nanoemulsion.

\section{Conclusions}

This study developed nanoemulsion systems using natural biopolymers, such as alginate and chitosan, for use as a bioactive ingredient delivery system, and investigated the physical properties of the fabricated nanoemulsion. The core material consisted of $\mathrm{OC}$, and the nanoemulsions were prepared using self-assembly methods, which have been previously successfully used to prepare nanoemulsions. The particle size of $\mathrm{SN}, \mathrm{AN}, \mathrm{CN}$, and CAN was $12.68 \pm 1.41$, $19.79 \pm 0.26,19.97 \pm 0.77$, and $16.69 \pm 0.43 \mathrm{~nm}$, respectively; therefore, we successfully manufactured stable nanoscale double- and triple-layer nanoemulsions that were $20 \mathrm{~nm}$ or lower. The Zeta potential values, which is an indicator of overall stability and physicochemical properties in nanoemulsion, of $\mathrm{AN}$ and $\mathrm{CN}$ were significantly higher than $\mathrm{SN}$ and CAN $(p<0.01)$. In conclusion, the double-layer nanoemulsions incorporated with alginate and chitosan can be expected to improve the stability of nanoemulsion, and this system hold promise for use in the production of functional foods containing bioactive ingredients.

Acknowledgments This study was supported by the Food Nanotechnology Development Project (2007), Ministry of Education, Science and Technology, Korea.

Open Access This article is distributed under the terms of the Creative Commons Attribution Noncommercial License which permits any noncommercial use, distribution, and reproduction in any medium, provided the original author(s) and source are credited.

\section{References}

Attwood, D., Mallon, C., Ktistis, G., \& Taylor, C. J. (1992). A study on factors influencing the droplet size in nonionic oil-in-water microemulsions. International Journal of Pharmaceutics, 88, 417-422.

Chen, H., Weiss, J., \& Shahidi, F. (2006a). Nanotechnology in nutraceuticals and functional foods. Food Technology, 60(3), 30-36.

Chen, L. Y., Remondetto, G. E., \& Subirade, M. (2006b). Food protein-based materials as nutraceutical delivery systems. Trends in Food Science and Technology, 17(5), 272-283.

Cho, Y. J., Kim, C. J., Kim, N. S., Kim, C. T., \& Park, B. (2008). Some cases in applications of nanotechnology to food and agricultural systems. Biochip Journal, 2(3), 183-185.

Choi, A. J., Kim, C. J., Cho, Y. J., Hwang, J. K., \& Kim, C. T. (2008). Solubilization of capsaicin and its nanoemulsion formation in the sonication and self-assembly methods. NSTI Nanotechnology Conference 2008, 4 June 2008, Boston, MA, USA.

GåserØd, O., SmiderØd, O., \& Skjåk-Braek, G. (1998). Microcapsules of alginate-chitosan-I A quantitative study of the interaction between alginate and chitosan. Biomaterials, 19, $1815-1825$.

GåserØd, O., SmiderØd, O., \& Skjåk-Braek, G. (1999). Microcapsules of alginate-chitosan- I I. A study of capsules stability and permeability. Biomaterials, 20, 773-783. 
Kim, C. T., Choi, A. J., Kim, C. J., \& Cho, Y. J. (2008). Preparation of food nanoemulsions containing bioactive ingredients and its application for use as nutraceutical delivery system. Nano Korea 2008, KINTEX, Kyeonggi, Korea.

Li, Y. Y., Chen, X. G., Liu, C. S., Cha, D. S., Park, H. J., \& Lee, C. M. (2007). Effect of the molecular mass and degree of substitution of oleoylchitosan on the structure, rheological properties, and formation of nanoparticles. Journal of Agricultural and Food Chemistry, 55(12), 4842-4847.

McClements, D. J., Decker, E. A., \& Weiss, J. (2007). Emulsion-based delivery systems for lipophilic bioactive components. Journal of Food Science, 72(8), R109-R124.

Neethirajan, S., \& Jayas, D. S. (2011). Nanotechnology for the food and bioprocessing industries. Food and Bioprocess Technology, 4, 39-47.
Nielloud, F., \& Marti-Mestres, G. (2000). Pharmaceutical emulsions and suspensions. New York: Marcel Dekker.

Seo, S. B., \& Toshio, K. (2002). Preparation of water soluble chitosan blendmer and their application to removal of heavy metal ions from wastewater. Macromolecular Research, 10(2), 103-107.

Voon, H. C., Bhat, R., Easa, A. M., Liong, M. T., \& Karim, A. A. (2010). Effect of addition of halloysite nanoclay and $\mathrm{SiO}_{2}$ nanoparticles on barrier and mechanical properties of bovine gelatin films. Food Bioprocess Technology. doi:10.1007/s11947010-0461-y.

Wang, L., Li, X., Zhang, G., Dong, J., \& Eastoe, J. (2007). Oil-inwater nanoemulsions for pesticide formations. Journal of Colloid and Interface Science, 314(1), 230-235.

Weiss, J., Takhistov, P., \& Mcclements, J. (2006). Functional materials in food nanotechnology. Journal of Food Science, 71(9), 107-116. 